\title{
HSP27 regulates TGF- $\beta$ mediated lung fibroblast differentiation through the Smad3 and ERK pathways
}

\author{
GANG WANG $^{1}$, HAO JIAO ${ }^{2}$, JUN-NIAN ZHENG ${ }^{1,3}$ and XIA SUN ${ }^{1}$ \\ ${ }^{1}$ Laboratory of Biological Cancer Therapy, Cancer Institute, Xuzhou Medical College; ${ }^{2}$ Department of Anesthesiology, \\ Affiliated Hospital of Xuzhou Medical College; ${ }^{3}$ Jiangsu Center for the Collaboration and Innovation of Cancer Biotherapy, \\ Cancer Institute, Xuzhou Medical College, Xuzhou, Jiangsu 221002, P.R. China
}

Received April 4, 2016; Accepted November 15, 2016

DOI: $10.3892 /$ ijmm.2016.2813

\begin{abstract}
Idiopathic pulmonary fibrosis (IPF) is a chronic lethal interstitial lung disease with unknown etiology. Recent studies have indicated that heat-shock protein 27 (HSP27) contributes to the pathogenesis of IPF through the regulation of epithelial-mesenchymal transition (EMT). However, the expression and role of HSP27 in fibroblasts during pulmonary fibrogenesis has not been investigated to date, at least to the best of our knowledge. In this study, we examined the expression of HSP27 in fibrotic lung tissue and fibroblasts from bleomycin (BLM)-challenged mice and human lung fibroblasts treated with transforming growth factor- $\beta$ (TGF- $\beta$ ). The results revealed that the expression of HSP27 was significantly increased in fibrotic lung tissue and fibroblasts from BLM-challenged mice. In vitro, TGF- $\beta$ stimulated HSP27 expression in and the differentiation of human lung fibroblasts. The knockdown of Smad3 expression or nuclear factor- $\kappa \mathrm{B}$ p65 subunit attenuated the TGF- $\beta$-induced increase in HSP27 expression and the differentiation of human lung fibroblasts. In addition, the knockdown of HSP27 expression attenuated the TGF- $\beta$-induced activation of ERK and Smad3, and inhibited the differentiation of human lung fibroblasts. On the whole, the findings of our study demonstrate that HSP27 expression is upregulated in lung fibroblasts during pulmonary fibrosis, and subsequently, HSP27 modulates lung fibroblast differentiation through the Smad3 and ERK pathways.
\end{abstract}

Correspondence to: Dr Xia Sun, Laboratory of Biological Cancer Therapy, Cancer Institute, Xuzhou Medical College, 84 West Huai-Hai Road, Xuzhou, Jiangsu 221002, P.R. China

E-mail: xsun711@123.com

Dr Jun-Nian Zheng, Jiangsu Center for the Collaboration and Innovation of Cancer Biotherapy, Cancer Institute, Xuzhou Medical College, 84 West Huai-Hai Road, Xuzhou, Jiangsu 221002, P.R. China E-mail: jnzheng@xzmc.edu.cn

Key words: heat-shock protein 27, fibroblast differentiation, pulmonary fibrosis, idiopathic pulmonary fibrosis

\section{Introduction}

Idiopathic pulmonary fibrosis (IPF) is a chronic, progressive interstitial lung disease (ILD), with the worst prognosis among all types of ILD (1). In the US, 128,100 individuals are affected by IPF, 48,000 new cases of IPF are diagnosed and 40,000 patients with IPF succumb to the disease annually $(2,3)$. Although there are some novel drugs available for the treatment IPF which have been approved in the US and Europe, the effectiveness of these drugs in the treatment of IPF remains limited (4). Pro- and anti-inflammatory cytokines, growth factors, reactive oxygen species (ROS) and lipid mediators have been implicated in the development of pulmonary fibrosis (5-7). However, to date, the mechanisms through which pathological changes progress in IPF remain unknown.Thus, the complete understanding of the pathogenesis of IPF remains to be achieved and this is a critical step in the development of effective therapeutic approaches.

Heat-shock protein 27 (HSP27) is a member of the small HSP family and is strongly induced by physiological and pathological stresses (8). Hsp27 is found highly expressed (9) and involved in tumorgenesis in various tissue and organs (10). Recent studies indicate that HSP27 is overexpressed in clusters between luminal epithelial cells and myofibroblasts in IPF lung tissue (11). Furthermore, a previous in vitro investigation demonstrated that HSP27 was directly related to the process of epithelial-mesenchymal transition (EMT) in lung mesothelial and epithelial cells (11). In addition, treatment with the HSP27 specific inhibitor, OGX-427, significantly ameliorated pleural/subpleural fibrosis in an animal model (11). However, the role and expression of HSP27 in lung fibroblasts under fibrotic conditions has not yet been investigated, at least to the best of our knowledge.

Bleomycin (BLM) has been extensively used to construct models of lung fibrosis in both mice and rats (12). In the mouse model of BLM-induced pulmonary fibrosis, transforming growth factor- $\beta$ (TGF- $\beta$ ) plays critical roles $(7,12)$. On the one hand, the expression of TGF- $\beta$ leads to the disruption of the homeostatic microenvironment, which is critical to promote fibroblast activation, migration, invasion and hyperplastic changes in the progression of IPF (7). In particular, lung fibroblast differentiation and accumulation induced by TGF- $\beta$ are the major causes of pulmonary fibrogenesis (13). Recently, HSP27 was proven to be essential for TGF- $\beta$-induced EMT 
features by in vitro study using human epithelial cell lines (11). Therefore, we hypothesized that HSP27 may be involved in the TGF- $\beta$-induced differentiation of lung fibroblasts.

In this study, we detected the expression of HSP27 during pulmonary fibrosis. We found that the expression of HSP27 was markedly upregulated in lung tissue and fibroblasts from BLM-challenged mice. Furthermore, we demonstrated that TGF- $\beta$-induced HSP27 expression was Smad3- and nuclear factor- $\kappa \mathrm{B}(\mathrm{NF}-\kappa \mathrm{B})-$ dependent in vitro. Additionally, the expression of HSP27 promoted TGF- $\beta$-induced fibroblast differentiation through the activation of Smad3 and ERK. By contrast, the depletion of HSP27 partially inhibited the activation of Smad3 and ERK, and thus prevented the differentiation of TGF- $\beta$-treated lung fibroblasts.

\section{Materials and methods}

Mouse model of experimental pulmonary fibrosis. As previously described (14,15), wild-type mice (C57BL/6J, male, 8 weeks old; $n=32$ ) were purchased from Vital River Laboratory Animal Technology (Beijing, China) and housed in a pathogen-free and light-controlled room (12 h light and $12 \mathrm{~h}$ dark) with free access to food and water. BLM sulfate from Hospira, Inc. (Lake Forest, IL, USA) was used to induce fibrosis. The mice were anesthetized (with a $3 \mathrm{ml} / \mathrm{kg}$ mixture of $25 \mathrm{mg} / \mathrm{kg}$ of ketamine in $2.5 \mathrm{ml}$ of xylazine), followed by treatment with either saline ( $\mathrm{n}=3-4$ mice) or BLM ( $\mathrm{n}=3-4$ mice) $(1.5 \mathrm{U} / \mathrm{kg}$ of body weight) in saline solution through an intratracheal (IT) injection. Twenty-one days post-BLM administration, the animals were sacrificed (by cervical dislocation following $\mathrm{CO}_{2}$ inhalation), and the lungs were removed for histological staining and the isolation of lung fibroblasts. All animal experiments were performed in accordance with protocols approved by the Animal Care and Use Committee of Xuzhou Medical College, Xuzhou, China.

Fluorescence staining. The lung tissues were fixed in formalin and then embedded in paraffin. Thereafter the samples were cut into 5- $\mu \mathrm{m}$-thick sections. After removing the paraffin, the mouse lung tissue sections (5- $\mu \mathrm{M}$-thick) were hydrated and washed in deionized water followed by block solution [Trisbuffered saline (TBS) with 1\% fetal bovine serum (FBS) $1 \mathrm{~h}]$ at room temperature, incubated with goat anti-HSP27 antibody and rabbit anti-fibronectin (FN) antibody (1:500 in the block solution; Santa Cruz Biotechnology, Inc., Santa Cruz, CA, USA) at $4^{\circ} \mathrm{C}$ overnight, and incubated with Alexa Fluor secondary antibodies (1:200 dilutions in blocking buffer) for $1 \mathrm{~h}$, followed by mounting; and examined under a Nikon Eclipse TE 2000-S fluorescence microscope with a $60 \mathrm{X}$ oil immersion objective lens. For human lung fibroblasts, the treated cells were fixed and permeabilized, then incubated with primary antibodies (1:200 dilutions in blocking buffer) for $1 \mathrm{~h}$ and washed 3 times, thereafter incubated with Alexa Fluor secondary antibodies (Alexa Fluor 594 for HSP27 and Alexa Fluor 488 for Fibronectin; Biolegend, San Diego, CA, USA; 1:200 dilutions in blocking buffer) for another $1 \mathrm{~h}$. The nuclei were counterstained with DAPI $(0.5 \mu \mathrm{g} / \mathrm{ml}$; SigmaAldrich, Inc., St. Louis, MO, USA). After washing, the slides were mounted on slides and examined under a fluorescence microscope (Olympus IX83; Olympus, Tokyo, Japan), as previously described $(16,17)$.
Isolation of primary mouse lung fibroblasts and cell culture. Primary mouse lung fibroblasts were isolated from the C57BL/6J mice treated with or without BLM as previously described (16), and the isolated cells were cultured in Dulbecco's modified Eagle's medium (DMEM) containing 10\% FBS for 14 days. The WI-38 cells (a human lung fibroblast cell line) was obtained from the American Type Culture Collection (ATCC, Manassas, VA, USA) and the cells were grown and maintained in 6-well plates with DMEM medium containing 10\% FBS.

Treatment with TGF- $\beta$ and $N F-\kappa B$ inhibitor. Recombinant human TGF- $\beta 1$ was obtained from Peprotech, Inc. (Rocky Hill, NJ, USA) and dissolved in phosphate-buffered saline (PBS) solution. Serum-starved lung fibroblasts ( $80 \%$ confluent) were treated with TGF- $\beta(5 \mathrm{ng} / \mathrm{ml})$ for $0-48 \mathrm{~h}$. NF- $\kappa$ B inhibitor was used as previously described (16). In detail, NF- $\kappa \mathrm{B}$ inhibitor (Bay 11-7082), which was obtained from Sigma-Aldrich, Inc., was applied to the lung fibroblasts at a final concentration of $10 \mu \mathrm{M}$ for $1 \mathrm{~h}$, as previously described (16). The NF- $\mathrm{BB}$ inhibitor was dissolved in DMSO; DMSO was used as a parallel control to eliminate the influence of DMSO itself. The cells were subsequently challenged with TGF- $\beta(5 \mathrm{ng} / \mathrm{ml})$ for $48 \mathrm{~h}$, and cell lysates ( $20 \mu \mathrm{g}$ protein) were subjected to western blot analysis.

Transfection with siRNA and shRNA. The scrambled RNA (scRNA), siRNA targeting human NF- $\mathrm{B}$ p65 subunit (si-p65) and siRNA targeting the human Smad3 (si-Smad3) smart pool, used in this study were from Santa Cruz Biotechnology, Inc. Vehicle shRNA (Veh shRNA) and shRNA targeting human HSP27 shRNA (HSP27 shRNA) were from Sigma-Aldrich, Inc. For target gene depletion, the WI-38 cells cultured on 6-well plates (50-60\% confluence) were transiently transfected with $200 \mathrm{nmol} / \mathrm{l}$ of siRNA (scRNA, si-p65 or si-Smad3), or transfected with $3 \mu \mathrm{g} /$ well of shRNA (Veh shRNA or HSP27 shRNA) using Attractene transfection reagent according to the manufacturer's instructions (Qiagen, Inc., Gaithersburg, MD, USA). After $48 \mathrm{~h}$, the cells were further treated with TGF- $\beta(5 \mathrm{ng} / \mathrm{ml})$ for a further $0-48 \mathrm{~h}$. Protein expression was analyzed by western blot analysis or under a fluorescence microscope, and the mRNA levels were analyzed by reverse transcription-quantitative PCR (RT-qPCR).

Western blot analysis. Sodium dodecyl sulfate-polyacrylamide gel electrophoresis (SDS-PAGE) and western blot analysis were performed as previously described $(16,18)$. The protease inhibitor cocktail tablets (EDTA-free complete) were from Roche Diagnostics (Indianapolis, IN, USA). Mouse anti- $\alpha$-smooth muscle actin ( $\alpha$-SMA) and anti- $\beta$-actin antibodies were from Sigma-Aldrich, Inc. Cell lysis buffer and rabbit anti-Smad3 antibody was from Cell Signaling Technology, Inc. (Danvers, MA, USA). Rabbit anti-FN and anti-glyceraldehyde 3-phosphate dehydrogenase (GAPDH) antibodies, mouse anti-p65 antibody and goat anti-HSP27 antibody were purchased from Santa Cruz Biotechnology, Inc. Horseradish peroxidase (HRP)-linked antimouse IgG and anti-rabbit IgG antibodies were obtained from Bio-Rad Laboratories, Inc. (Hercules, CA, USA). Briefly, cell lysates were prepared in lysis buffer containing EDTA-free complete protease inhibitors, followed by centrifugation at $10,000 \times \mathrm{g}$ for $10 \mathrm{~min}$, boiled with Laemmli sample buffer 
A
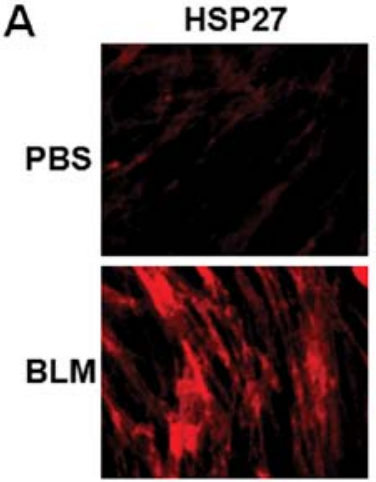

B

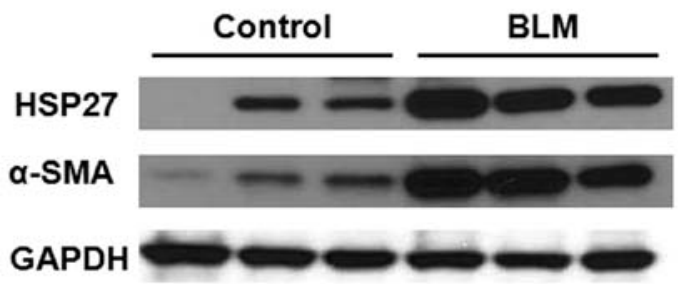

FN
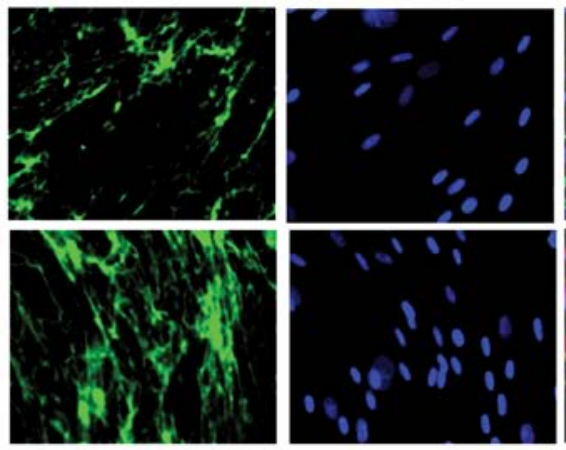

DAPI
Merge

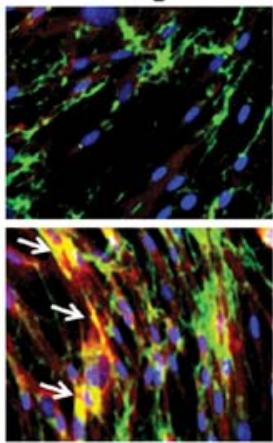

C

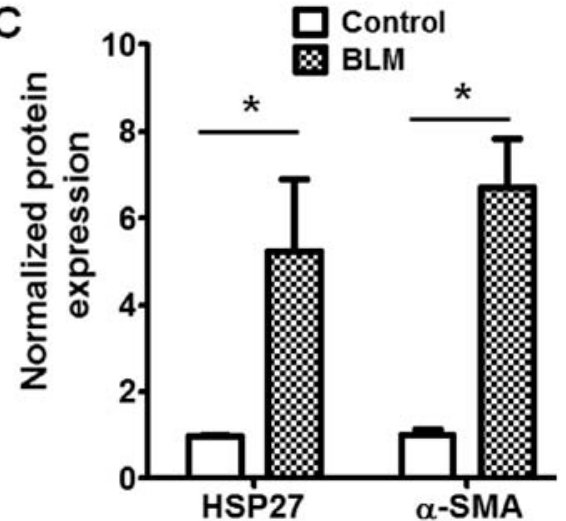

Figure 1. Heat-shock protein 27 (HSP27) expression in lung tissue from mice. (A) Representative immunofluorescence staining of HSP27 (green), fibronectin (FN) (red) and DAPI (blue) in mouse lung tissue from mice with/without bleomycin (BLM) challenge. Arrows indicate the expression of HSP27 co-localized with FN (yellow) in fibrotic foci. (B) Representative western blots and (C) the quantification of the expression of HSP27 and $\alpha$-smooth muscle actin ( $\alpha$-SMA) in lung tissue from mice with or without BLM challenge. Data are expressed as the means \pm SEM. ${ }^{*} \mathrm{P}<0.05$, $\mathrm{n}=4$ experiments.

for 5 min, separated on 10 or 4-20\% SDS-PAGE, transferred onto PVDF membranes, and blocked with TBST containing $5 \%$ BSA prior to incubation with primary antibodies $(1: 1,000$ dilution) overnight, and secondary antibodies (1:2,000 dilutions) for $2 \mathrm{~h}$ at room temperature. The blots were developed and quantified using ImageQuant 5.2 software (Molecular Dynamics, Sunnyvale, CA, USA).

RNA isolation and real-time PCR. Total RNA was isolated from the cells using TRIzol reagent (Life Technology, Rockville, MD,USA), and RNA ( $1 \mu \mathrm{g})$ was reversed transcribed using the cDNA synthesis kit (Bio-Rad Laboratories, Inc.). Quantitative PCR (qPCR) was performed as previously described $(14,16)$. Briefly, qPCR detection was performed using the ABI 7500 system (Life Technologies, Grand Island, NY, USA) with the SYBR-Green supermix kit (Bio-Rad Laboratories, Inc.). According to the manufacturer's instructions, the running parameters were set as denaturation at $95^{\circ} \mathrm{C}$ for $3 \mathrm{~min}, 40$ cycles of denaturation at $95^{\circ} \mathrm{C}$ for $15 \mathrm{sec}$ and annealing/extension at $60^{\circ} \mathrm{C}$ for $1 \mathrm{~min}$, finally the melt curve analysis was set as $95^{\circ} \mathrm{C}$ for $15 \mathrm{sec}, 60^{\circ} \mathrm{C}$ for $1 \mathrm{~min}$ and $95^{\circ} \mathrm{C}$ for $15 \mathrm{sec}$. For human $\mathrm{Smad} 3$, the primer sequence was forward, 5'-TGGACGCAGGTTCTCCAAAC-3' and reverse, 5'-CCGGCTCGCAGTAGGTAAC-3'; for human GAPDH, the primer sequence was forward, 5'-TGTGGGCATCAATG GATTTGG-3' and reverse, 5'-ACACCATGTATTCCGG GTCAAT-3'. GAPDH was used as a housekeeping gene to normalize the expression levels as previously described (14).
Statistical analysis. Primary data from at least 3 independent experiments are expressed as the means \pm SEM, and subjected to statistical analysis using the two-tailed Student's t-test or one-way ANOVA. Values of $\mathrm{P}<0.05$ were considered to indicate statistically significant differences $(19,20)$.

\section{Results}

BLM challenge increases HSP27 expression in mouse lung tissue. The role of HSP27 in the etiology of lung fibrosis is elusive. In this study, we examined the expression of HSP27 in lung tissue from C57BL/6J mice challenged with BLM by IT injection (male, 8 weeks old) or treated with saline. At 21 days post-challenge, lung tissues were isolated from the mice and and used for immunofluorescence staining for HSP27 and $\mathrm{FN}$ as described in the Materials and methods. As shown in Fig. 1A, the expression of HSP27 was significantly increased and co-localized with FN, a marker of fibrogenesis, in the lung fibrotic foci from the BLM-challenged mice. The resutls of western blot analysis also indicated that HSP27 expression was markedly increased in the lung tissue from the BLM-challenged mice (Fig. 1B and C).

HSP27 expression in lung fibroblasts. We then examined the expression of HSP27 in lung fibroblasts from the control and BLM-challenged mice. As shown in Fig. 2, the expression levels of HSP27, as well as that of bio-markers of fibroblast differentiation ( $\alpha$-SMA and FN), were markedly higher in the 
A

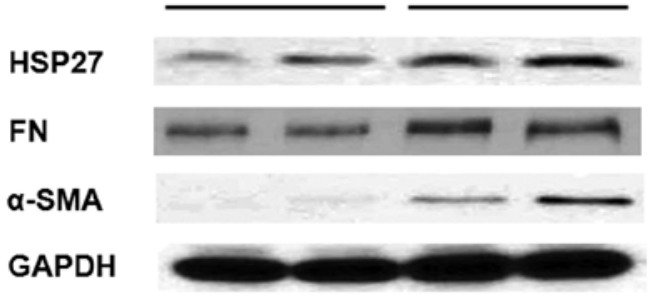

B

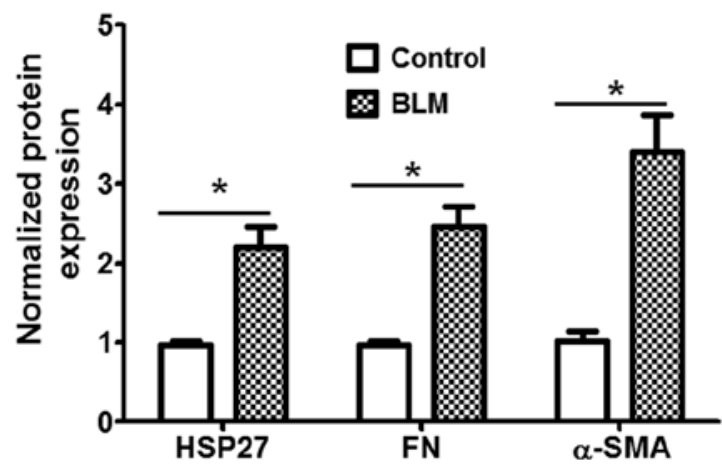

Figure 2. Expression of heat-shock protein 27 (HSP27) in lung fibroblasts from mice with or without bleomycin (BLM) challenge. C57BL/6J mice (male, 8 weeks old) were treated with either saline or challenged with BLM (1.5 U/kg of body weight, $\sim 0.03$ units/animal) by IT injection. Primary lung fibroblasts isolated from mice with or without BLM challenge (21 days post-challenge) were used for western blot analysis as described in the Materials and methods. (A) Representative western blots and (B) quantification of the expression of HSP27, fibronectin (FN) and $\alpha$-smooth muscle actin $(\alpha-\mathrm{SMA})$ in lung fibroblasts isolated from mice with or without BLM challenge. The expression of HSP27, FN and $\alpha$-SMA was quantified and normalized to that of GAPDH. Data are expressed as the means \pm SEMs of 3 independent experiments. ${ }^{*} \mathrm{P}<0.05$.

A

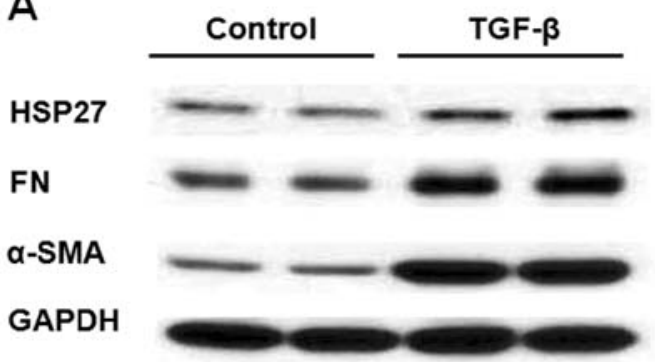

C

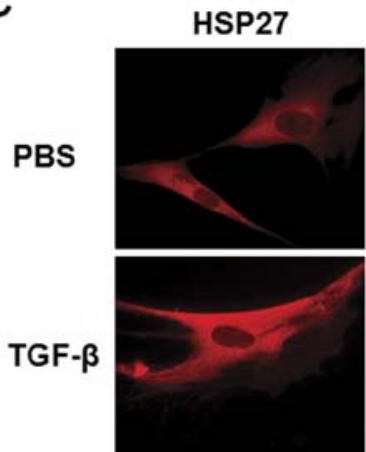

FN
B
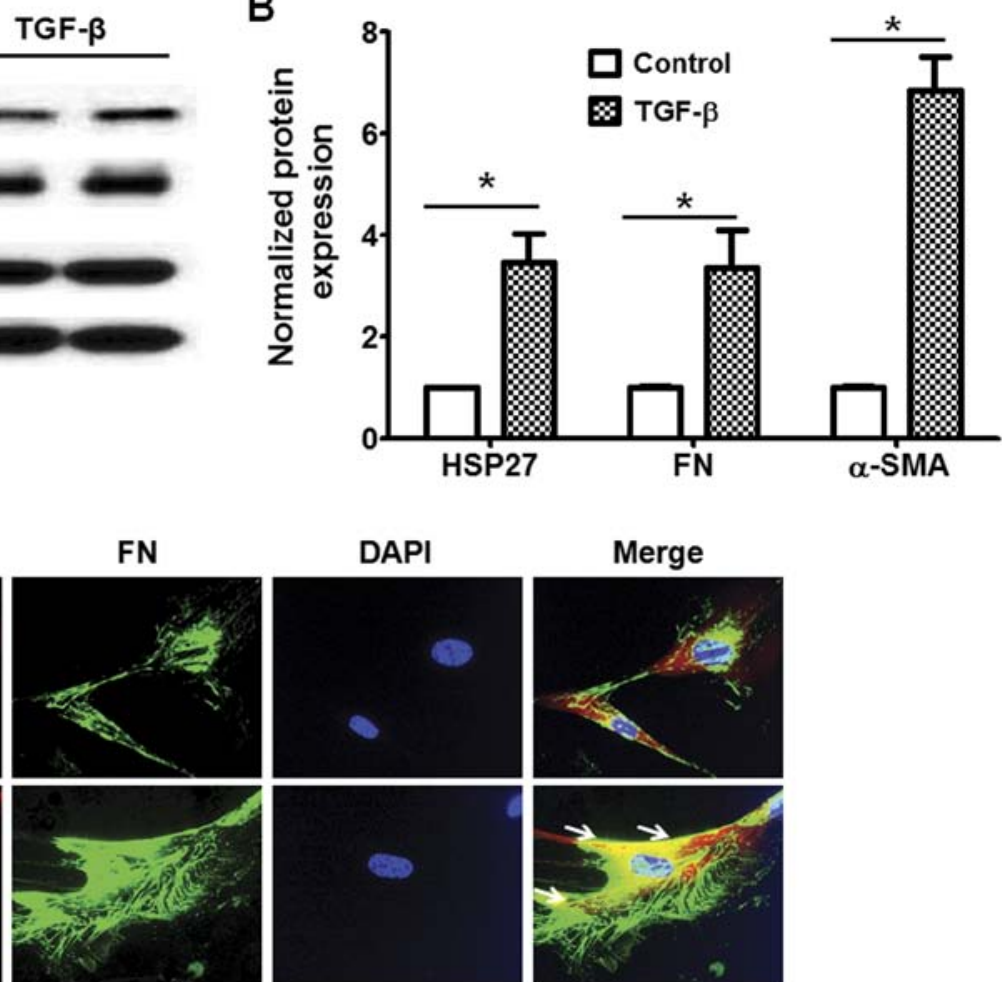

DAPI
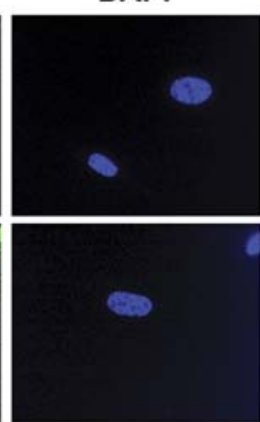

Merge

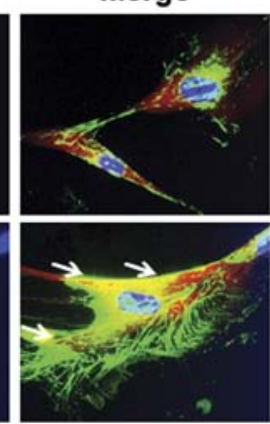

Figure 3. Transforming growth factor- $\beta$ (TGF- $\beta$ ) induces heat-shock protein 27 (HSP27) expression in human lung fibroblasts. Human lung fibroblasts (WI-38, $\sim 80 \%$ confluent $)$ were starved for $24 \mathrm{~h}$, followed by challenge with TGF- $\beta(5 \mathrm{ng} / \mathrm{ml})$ for a further $48 \mathrm{~h}$. Cell lysates were used in western blot analysis as described in the Materials and methods. The intensity of bands with anti-fibronectin (FN), anti- $\alpha$-smooth muscle actin ( $\alpha$-SMA) and anti-HSP27 antibodies were quantified and normalized to GAPDH. (A) Representative western blots and (B) quantification of the expression of HSP27, FN and $\alpha$-SMA in human lung fibroblasts with or without TGF- $\beta$ challenge. Data are expressed as the means \pm SEM of 3 independent experiments. ${ }^{*}<<0.05$. (C) Representative immunofluorescence staining for HSP27 (green), FN (red) and DAPI (blue) in human lung fibroblast with or without TGF- $\beta$ challenge $(5 \mathrm{ng} / \mathrm{ml}, 48 \mathrm{~h})$. White arrows indicated the increased expression of HSP27 and FN in fibroblasts post TGF- $\beta$ challenge.

lung fibroblasts from BLM-challenged mice than in those from the control mice. These findings suggest that HSP27 expression in lung fibroblasts may be associated with fibroblast differentiation under fibrotic conditions.

TGF- $\beta$ promotes HSP27 expression in human lung fibroblasts. TGF- $\beta$ is a key factor for fibroblast differentiation through the regulation of the expression of various genes during pulmonary fibrosis (21). Thus, we then investigated whether HSP27 expression is regulated by TGF- $\beta$ in lung fibroblasts. As shown in Fig. $3 \mathrm{~A}$ and $\mathrm{B}$, the TGF- $\beta$ challenge $(5 \mathrm{ng} / \mathrm{ml}, 48 \mathrm{~h}$ ) markedly increased HSP27 expression and promoted fibroblast differentiation. Furthermore, we confirmed the expression of HSP27 by immunofluorescence staining. Examination under 
A

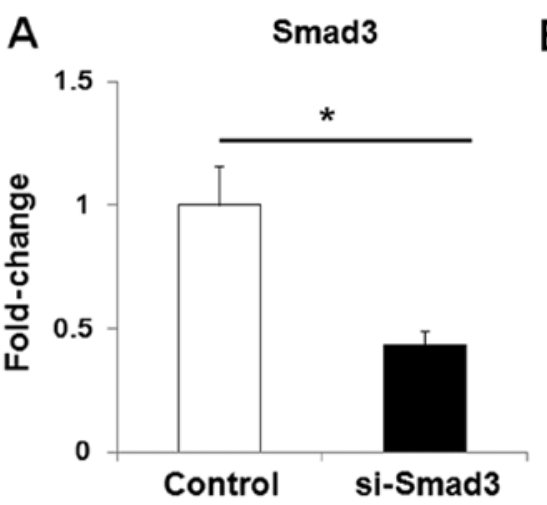

C

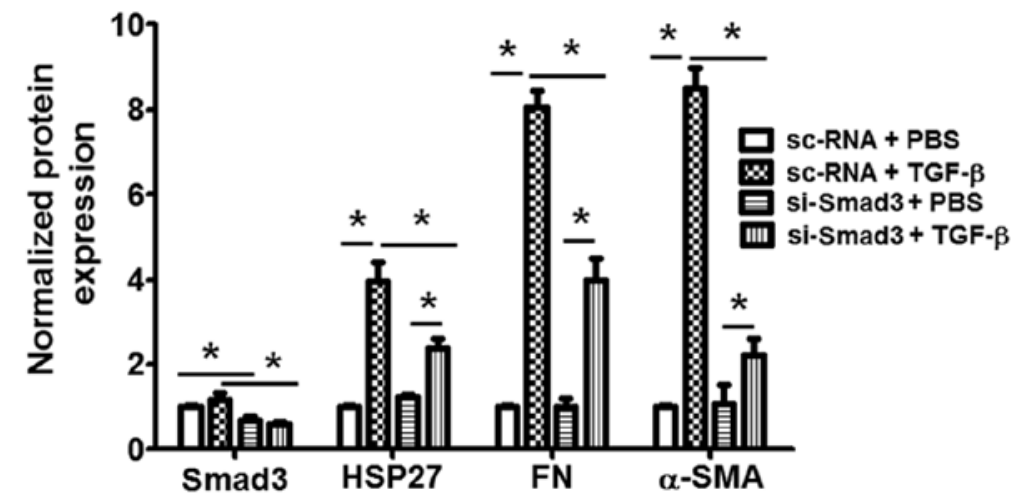

B

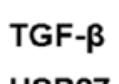

HSP27

FN

a-SMA

Smad3

GAPDH

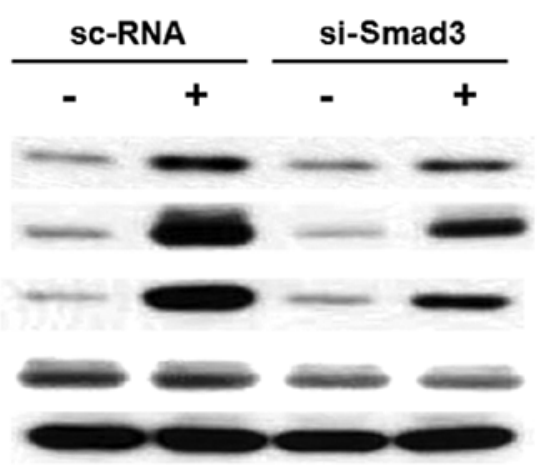

Figure 4. Knockdown of Smad3 attenuates transforming growth factor- $\beta$ (TGF- $\beta$ ) induced heat-shock protein 27 (HSP27) expression in human lung fibroblasts. WI-38 cells with transient transfection of scRNA and Smad3 siRNA ( $200 \mathrm{nmol} / \mathrm{l})$ as described in the Materials and methods followed by TGF- $\beta(5 \mathrm{ng} / \mathrm{ml}, 48 \mathrm{~h})$ challenge. (A) The mRNA level of Smad3 was analyzed by RT-qPC, (B) the protein expression of Smad3, HSP27, fibronectin (FN) and $\alpha$-smooth muscle actin ( $\alpha$-SMA) was examined by western blot analysis. (B) Representative western blots and (C) quantification of the expression of Smad3, HSP27, FN and $\alpha$-SMA in human lung fibroblasts. Data are expressed as the means \pm SEM of 3 independent experiments. ${ }^{*} \mathrm{P}<0.05$.

a fluorescence microscope also indicated that the TGF- $\beta$ challenge upregulated the expression of HSP27 and FN in human lung fibroblasts (Fig. 3C).

TGF- $\beta$ induces HSP27 expression through the Smad and $N F-\kappa B$ pathways. To further determine the molecular mechanisms through which TGF- $\beta$ regulates HSP27 expression in lung fibroblasts, we knocked down the expression of Smad3 or the NF- $\mathrm{B}$ p65 subunit, which are the key transcription factors in TGF- $\beta$-challenged lung fibroblasts. Transfection with si-Smad3 significantly decreased the mRNA (Fig. 4A) and protein expression (Fig. $4 \mathrm{~B}$ and $\mathrm{C}$ ) of Smad3 in the human lung fibroblasts, and also inhibited the TGF- $\beta$-induced HSP27 expression in the human lung fibroblasts (Fig. 4B and C). Similarly, the knockdown of the p65 subunit by transfection with si-p65 also markedly inhibited the TGF- $\beta$-induced HSP27 expression and the differentiation of human lung fibroblasts (Fig. 5A and B). Additionally, treatment with $\mathrm{NF}-\kappa \mathrm{B}$ inhibited the TGF- $\beta$-induced increase in HSP27 expression and the differentiation of human lung fibroblasts (Fig. 5C and D). Taken together, these data indicated that TGF- $\beta$ induced HSP27 expression through the Smad 3 and NF- $\mathrm{NB}$ pathways in human lung fibroblasts.

HSP 27 regulates TGF- $\beta$-induced human lung fibroblast differentiation via the regulation of Smad and ERK activation. We also examined the role of HSP27 in TGF- $\beta$-induced fibroblast differentiation. As shown in Fig. 6, transfection with HSP27 shRNA significantly decreased the expression of HSP27 in human lung fibroblasts, and also inhibited the TGF- $\beta$-induced differentiation of lung fibroblasts (Fig. 6). The TGF- $\beta$-induced activation of Smad 3 and ERK are critical for lung fibroblast differentiation during pulmonary fibrogenesis $(6,14)$. We then further examined whether HSP27 regulates the TGF- $\beta$-induced activation of the Smad and ERK signaling pathways in human lung fibroblasts. As shown in Fig. 7, the knockdown of HSP27 markedly blocked the TGF- $\beta$-induced activation of Smad3 and ERK. Additionally, the role of HSP27 in the TGF- $\beta$-induced activation of Akt, p38 and JNK was examined studied. However, the knockdown of HSP27 had shown no effect on the TGF- $\beta$-induced activation of Akt, p38 and JNK (data not shown). Taken together, these data indicate that HSP27 regulates TGF- $\beta$-induced human lung fibroblast differentiation through the regulation of Smad3 and ERK activation.

\section{Discussion}

IPF is a fatal fibrotic disease with a disconcerting survival rate, and predominantly occurs in middle-aged and older individuals (22). Some therapeutic progress has been over the past few years; however, this has been proven to be uneffective. Pirfenidone, an orally available pyridine derivative, was approved for the treatment of IPF in $2014(23,24)$. In models of BLM-induced lung fibrosis, treatment with pirfenidone has been shown to inhibit the upregulation of HSP47 expression in lung 
A

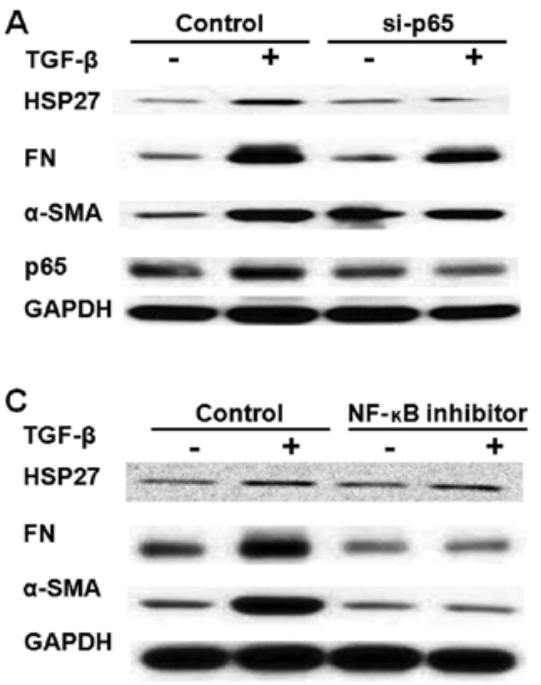

B
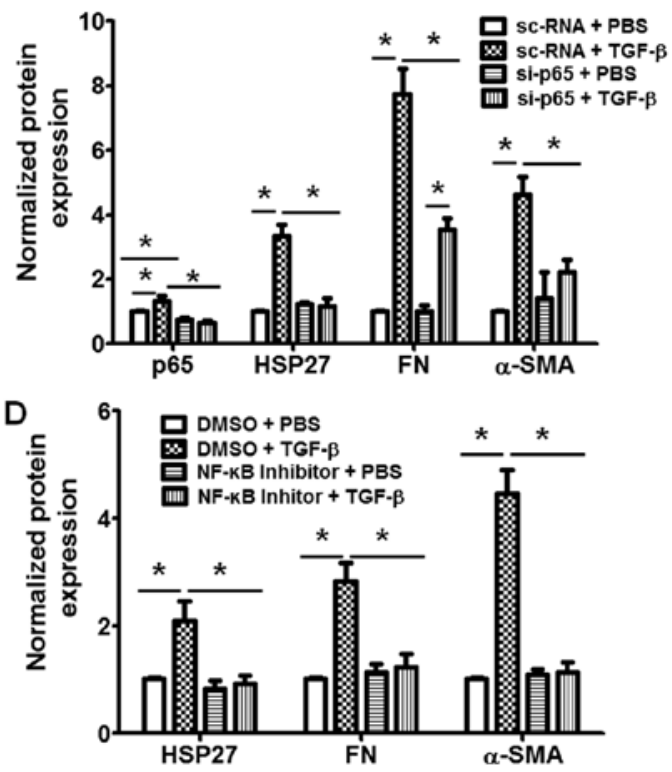

Figure 5. Transforming growth factor- $\beta$ (TGF- $\beta$ ) induces heat-shock protein 27 (HSP27) expression in human lung fibroblasts through the NF- $\mathrm{BB}$ pathway. (A and B) Knockdown of NF- $\mathrm{kB}$ p65 subunit attenuates TGF- $\beta$ induced HSP27 expression in human lung fibroblast. (A) Representative western blots and (B) quantification of the expression of p65, HSP27, fibronectin (FN) and $\alpha$-smooth muscle actin ( $\alpha$-SMA) in human lung fibroblasts. (C and D) NF- $\mathrm{BB}$ inhibitor attenuates TGF- $\beta$-induced expression of HSP27 in human lung fibroblasts. (C and D) Inhibition of NF- $\kappa$ B pathway attenuates TGF- $\beta$ induced HSP27 expression in human lung fibroblast. WI-38 cells with pre-treated with the vehicle solution (control; DMSO) and NF- $\mathrm{kB}$ inhibitor (Bay 11-7082, 10 $\mu \mathrm{M}, 1 \mathrm{~h}$ ) followed by TGF- $\beta(5 \mathrm{ng} / \mathrm{ml}, 48 \mathrm{~h}$ ) challenge. (C) Representative western blots and (D) quantification of the expression of HSP27, FN and $\alpha$-SMA in human lung fibroblasts with or without TGF- $\beta$ challenge. Data are expressed as the means \pm SEM of 3 independent experiments. ${ }^{*} \mathrm{P}<0.05$.

A

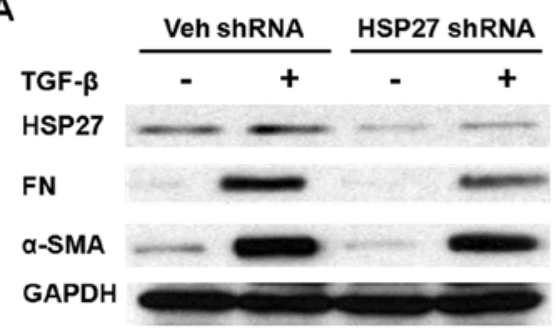

B

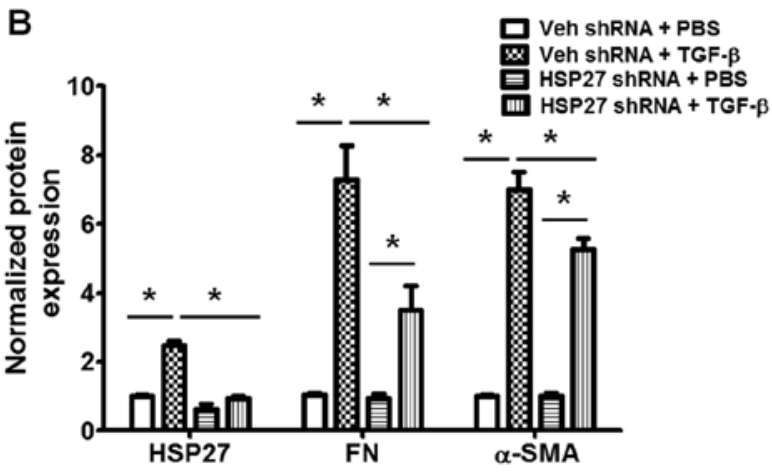

Figure 6. Knockdown of heatshock protein 27 (HSP27) expression attenuates transforming growth factor- $\beta$ (TGF- $\beta$ )-induced differentiation of human lung fibroblasts. WI-38 cells with transient transfection of vehicle shRNA and HSP27 shRNA ( $3 \mu \mathrm{g} / \mathrm{well}$, in 6-well plate) as described in the Materials and methods followed by TGF- $\beta(5 \mathrm{ng} / \mathrm{ml}, 0-48 \mathrm{~h}$ ) challenge. (A) Representative western blots and (B) quantification of the expression of HSP27, fibronectin (FN), and $\alpha$-smooth muscle actin $\left(\alpha\right.$-SMA) in human lung fibroblasts. Data are expressed as the means \pm SEM of 3 independent experiments. ${ }^{*} \mathrm{P}<0.05$.

A

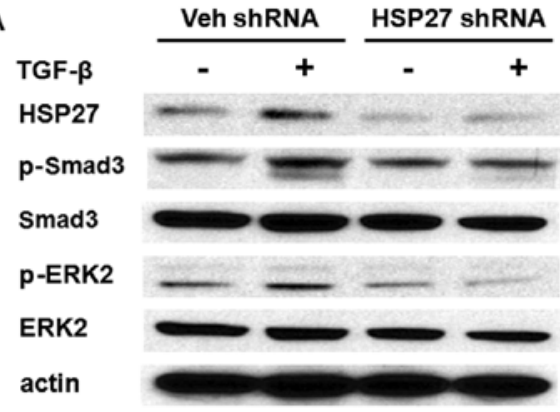

B

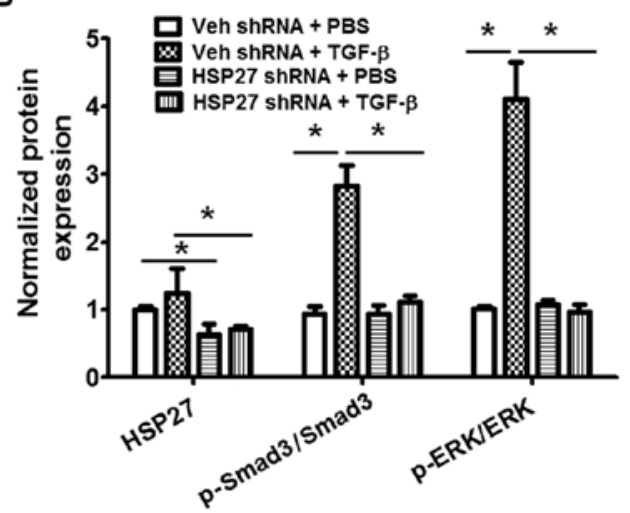

Figure 7. Knockdown heatshock protein 27 (HSP27) expression attenuates transforming growth factor- $\beta$ (TGF- $\beta$ )-induced activation of Smad 3 and ERK in human lung fibroblasts. WI-38 cells with transient transfection of vehicle shRNA and HSP27 shRNA ( $3 \mu \mathrm{g} /$ well, in 6-well plate) as described in the 'Materials and methods' followed by TGF- $\beta(5 \mathrm{ng} / \mathrm{ml}, 0-1 \mathrm{~h}$ ) challenge. (A) Representative western blots and (B) quantification of the expression of HSP27, p-ERK/ERK, and $\mathrm{p}-\mathrm{Smad} 3 / \mathrm{Smad} 3$ in human lung fibroblasts. Data are expressed as the means \pm SEMs of 3 independent experiments. ${ }^{*} \mathrm{P}<0.05$. 
epithelial cells and fibroblasts (25-27). Similar to HSP27, HSP47 is also a member of the small HSP family (28). Treatment with pirfenidone has been shown to attenuate pulmonary fibrosis in animal models $(27,29,30)$. Clinical trials with IPF patients have also indicated that pirfenidone is a potent anti-fibrotic, anti-inflammatory and antioxidant drug during pulmonary fibrogenesis $(4,24,31)$. These studies suggest that the HSP family of proteins play critical roles in IPF, and thus, understanding the role of the HSP family of proteins may be essential to the identification and development of novel therapeutic strategies for IPF.

HSP27 has been proven to participate in the pathogenesis of pulmonary fibrosis, and treatment with HSP27 inhibitors has been shown to attenuate bleomycin-induced pulmonary fibrosis by inhibiting epithelial-mesothelial transition in an animal model (11). In in vitro investigations with a human mesothelium cell line, Met-5A, it was found that through interactions with Snail and via the blocking of its proteasomal degradation, HSP27 accelerated EMT (11). It is possible that the HSP27-related EMT process is not limited to fibrogenesis, but may also be involved in other diseases in which EMT occurs. However, the expression and role of HSP27 in lung fibroblasts during pulmonary fibrosis remain elusive. In the present study, we isolated lung fibroblasts from mice with or without BLM-induced pulmonary fibrosis, and investigated the expression and role of HSP27 in lung fibroblasts during pulmonary fibrogenesis. Similar to the findings of a previous study, our data also indicated that the expression of HSP27 was markedly increased in experimental fibrotic lung tissue (Fig. 1). Of note, the expression of HSP27, as well as that of lung fibroblast differentiation protein markers ( $\mathrm{FN}$ and $\alpha$-SMA), in the primary lung fibroblasts from mice with pulmonary fibrosis was markedly higher than that in fibroblasts from the control mice (Fig. 2). In human lung fibroblasts, treatment with TGF- $\beta$ also markedly increased the expression of HSP27 in fibroblasts. Taken together, our data indicate that HSP27 may also be involve in fibroblast differentiation during lung fibrosis.

Studies have proven that TGF- $\beta$ is a key factor to promote lung fibroblast differentiation through Smad-dependent and -independent pathways (13). Additionally, TGF- $\beta$ is also involved in a crosstalk with wnt/ $\beta$-catenin, sphingosine 1 phospate, lysophosphatidic acid and NF- $\kappa B$ pathways to induce pro- or anti-fibrotic gene expression during pulmonary fibrogenesis $(5,14,15,32,33)$. To investigate the mechanisms through which TGF- $\beta$ induces HSP27 expression in lung fibroblasts, we knocked down the expression of Smad3 or NF- $\mathrm{KB}$ p65 subunit and examined the expression of HSP27 in TGF- $\beta$-treated cells. A previous study suggested that the depletion of HSP27 in epithelial cells significantly inhibited TGF- $\beta$-induced EMT and suppressed the expression of Snail, which is a subfamily of the zinc finger transcription factor that represses the transcription of the adhesion molecule, E-cadherin, and thus inhibits EMT (34). Similarly, this study indicated that the knockdown of HSP 27 markedly blocked the TGF- $\beta$-induced activation of Smad3 and ERK, and inhibited the expression of FN and $\alpha$-SMA in human lung fibroblasts (Figs. 6 and 7). Our findings indicated that TGF- $\beta$ induced HSP27 expression through the activation of the Smad3 and NF- $\mathrm{BB}$ pathways; furthermore, HSP27 expression also synergistically regulated the TGF- $\beta$ induced differentiation of human lung fibroblasts through the regulation of the Smad3 and ERK signaling pathways.
In conclusion, in this study, we demonstrated that HSP27 expression increased in lung fibrotic foci, and TGF- $\beta$ increased HSP27 expression through the activation of the Smad3 and NF- $\mathrm{kB}$ pathways. HSP27 regulated the TGF- $\beta$-induced activation of lung fibroblasts during pulmonary fibrogenesis.

\section{Acknowledgements}

This study was supported by the China National Natural Science Foundation (grant nos. 81400047 and 81400055) and the Natural Science Foundation of Jiangsu Province (grant nos. BK20150213 and BK20140242). The funders had no role in the study design, data collection and analysis, decision to publish, or preparation of the manuscript.

\section{References}

1. Zibrak JD and Price D: Interstitial lung disease: Raising the index of suspicion in primary care. NPJ Prim Care Respir Med 24: 14054, 2014.

2. Raghu G, Weycker D, Edelsberg J, Bradford WZ and Oster G: Incidence and prevalence of idiopathic pulmonary fibrosis. Am J Respir Crit Care Med 174: 810-816, 2006.

3. Esposito DB, Lanes S, Donneyong M, Holick CN, Lasky JA, Lederer D, Nathan SD, O'Quinn S, Parker J and Tran TN: Idiopathic pulmonary fibrosis in US automated claims: Incidence, prevalence and algorithm validation. Am J Respir Crit Care Med 192: 1200-1207, 2015.

4. Azuma A: Pirfenidone: Antifibrotic agent for idiopathic pulmonary fibrosis. Expert Rev Respir Med 4: 301-310, 2010.

5. Rancoule C, Pradère JP, Gonzalez J, Klein J, Valet P, Bascands JL, Schanstra JP and Saulnier-Blache JS: Lysophosphatidic acid-1receptor targeting agents for fibrosis. Expert Opin Investig Drugs 20: 657-667, 2011.

6. Huang LS and Natarajan V: Sphingolipids in pulmonary fibrosis. Adv Biol Regul 57: 55-63, 2015.

7. Wynn TA: Integrating mechanisms of pulmonary fibrosis. J Exp Med 208: 1339-1350, 2011.

8. Shashidharamurthy R, Koteiche HA, Dong J and McHaourab HS: Mechanism of chaperone function in small heat shock proteins: Dissociation of the HSP27 oligomer is required for recognition and binding of destabilized T4 lysozyme. J Biol Chem 280: 5281-5289, 2005.

9. Hadaschik BA, Jackson J, Fazli L, Zoubeidi A, Burt HM, Gleave ME and So AI: Intravesically administered antisense oligonucleotides targeting heat-shock protein-27 inhibit the growth of non-muscle-invasive bladder cancer. BJU Int 102: 610-616, 2008.

10. Kamada M, So A, Muramaki M, Rocchi P, Beraldi E and Gleave M: Hsp27 knockdown using nucleotide-based therapies inhibit tumor growth and enhance chemotherapy in human bladder cancer cells. Mol Cancer Ther 6: 299-308, 2007.

11. Wettstein G, Bellaye PS, Kolb M, Hammann A, Crestani B, Soler P, Marchal-Somme J, Hazoume A, Gauldie J, Gunther A, et al: Inhibition of HSP27 blocks fibrosis development and EMT features by promoting Snail degradation. FASEB J 27: 1549-1560, 2013.

12. Della Latta V, Cecchettini A, Del Ry S and Morales MA: Bleomycin in the setting of lung fibrosis induction: From biological mechanisms to counteractions. Pharmacol Res 97: 122-130, 2015.

13. Wolters PJ, Collard HR and Jones KD: Pathogenesis of idiopathic pulmonary fibrosis. Annu Rev Pathol 9: 157-179, 2014.

14. Huang LS, Fu P, Patel P, Harijith A, Sun T, Zhao Y, Garcia JG, Chun J and Natarajan V: Lysophosphatidic acid receptor-2 deficiency confers protection against bleomycin-induced lung injury and fibrosis in mice. Am J Respir Cell Mol Biol 49: 912-922, 2013.

15. Huang LS, Berdyshev E, Mathew B, Fu P, Gorshkova IA, He D, Ma W, Noth I, Ma SF, Pendyala S, et al: Targeting sphingosine kinase 1 attenuates bleomycin-induced pulmonary fibrosis. FASEB J 27: 1749-1760, 2013.

16. Sun X, Chen E, Dong R, Chen W and Hu Y: Nuclear factor (NF)- $\mathrm{kB}$ p65 regulates differentiation of human and mouse lung fibroblasts mediated by TGF- $\beta$. Life Sci 122: 8-14, 2015. 
17. Huang LS, Mathew B, Li H, Zhao Y, Ma SF, Noth I, Reddy SP, Harijith A, Usatyuk PV, Berdyshev EV, et al: The mitochondrial cardiolipin remodeling enzyme lysocardiolipin acyltransferase is a novel target in pulmonary fibrosis. Am J Respir Crit Care Med 189: 1402-1415, 2014.

18. Usatyuk PV, Burns M, Mohan V, Pendyala S, He D, Ebenezer DL, Harijith A, Fu P, Huang LS, Bear JE, et al: Coronin 1B regulates S1P-induced human lung endothelial cell chemotaxis: Role of PLD2, protein kinase C and Rac1 signal transduction. PLoS One 8: e63007, 2013.

19. Huang LS, Kim MR and Sok D-E: Enzymatic reduction of polyunsaturated lysophosphatidylcholine hydroperoxides by glutathione peroxidase-1. Eur J Lipid Sci Technol 111: 584-592, 2009.

20. HuangLS,Hung ND,SokDEandKimMR:Lysophosphatidylcholine containing docosahexaenoic acid at the sn-1 position is anti-inflammatory. Lipids 45: 225-236, 2010.

21. Midgley AC, Rogers M, Hallett MB, Clayton A, Bowen T, Phillips AO and Steadman R: Transforming growth factor- $\beta 1$ (TGF- $\beta 1$ )-stimulated fibroblast to myofibroblast differentiation is mediated by hyaluronan (HA)-facilitated epidermal growth factor receptor (EGFR) and CD44 co-localization in lipid rafts. J Biol Chem 288: 14824-14838, 2013.

22. Morell F: Idiopathic pulmonary fibrosis: Importance of accurate diagnosis and treatment. Arch Bronconeumol 49: 319-320, 2013

23. Antoniou KM, Tomassetti S, Tsitoura E and Vancheri C: Idiopathic pulmonary fibrosis and lung cancer: A clinical and pathogenesis update. Curr Opin Pulm Med 21: 626-633, 2015.

24. Aravena C, Labarca G, Venegas C, Arenas A and Rada G: Pirfenidone for idiopathic pulmonary fibrosis: A systematic review and meta-analysis. PLoS One 10: e0136160, 2015.

25. Hisatomi K, Mukae H, Sakamoto N, Ishimatsu Y, Kakugawa T, Hara S, Fujita H, Nakamichi S, Oku H, Urata Y, et al: Pirfenidone inhibits TGF- $\beta 1$-induced over-expression of collagen type I and heat shock protein 47 in A549 cells. BMC Pulm Med 12: 24 2012.
26. Nakayama S, Mukae H, Sakamoto N, Kakugawa T, Yoshioka S, Soda $\mathrm{H}$, Oku H, Urata Y, Kondo T, Kubota H, et al: Pirfenidone inhibits the expression of HSP47 in TGF-betal-stimulated human lung fibroblasts. Life Sci 82: 210-217, 2008.

27. Kakugawa T, Mukae H, Hayashi T, Ishii H, Abe K, Fujii T, Oku H, Miyazaki M, Kadota J and Kohno S: Pirfenidone attenuates expression of HSP47 in murine bleomycin-induced pulmonary fibrosis. Eur Respir J 24: 57-65, 2004.

28. Razzaque MS, Le VT and Taguchi T: Heat shock protein 47 and renal fibrogenesis. Contrib Nephrol 148: 57-69, 2005.

29. Schaefer CJ, Ruhrmund DW, Pan L, Seiwert SD and Kossen K: Antifibrotic activities of pirfenidone in animal models. Eur Respir Rev 20: 85-97, 2011.

30. Seifirad S, Keshavarz A, Taslimi S, Aran S, Abbasi H and Ghaffari A: Effect of pirfenidone on pulmonary fibrosis due to paraquat poisoning in rats. Clin Toxicol (Phila) 50: 754-758, 2012

31. Raghu G, Johnson WC, Lockhart D and Mageto Y: Treatment of idiopathic pulmonary fibrosis with a new antifibrotic agent, pirfenidone: Results of a prospective, open-label phase II study. Am J Respir Crit Care Med 159: 1061-1069, 1999.

32. Chilosi M, Poletti V, Zamò A, Lestani M, Montagna L, Piccoli P, Pedron S, Bertaso M, Scarpa A, Murer B, et al: Aberrant Wnt/beta-catenin pathway activation in idiopathic pulmonary fibrosis. Am J Pathol 162: 1495-1502, 2003.

33. Tager AM, LaCamera P, Shea BS, Campanella GS, Selman M, Zhao Z, Polosukhin V, Wain J, Karimi-Shah BA, Kim ND, et al: The lysophosphatidic acid receptor LPA1 links pulmonary fibrosis to lung injury by mediating fibroblast recruitment and vascular leak. Nat Med 14: 45-54, 2008.

34. Lamouille S, Xu J and Derynck R: Molecular mechanisms of epithelial-mesenchymal transition. Nat Rev Mol Cell Biol 15: 178-196, 2014. 